\title{
La Motivación en la Resolución de Problemas Aritmético-algebraicos. Un Estudio con Alumnado de Educación Secundaria
}

\author{
Javier Gasco Txabarri
}

$\mathbf{y}$

\section{José Domingo Villarroel Villamor}

Didáctica de la Matemática y de las Ciencias Experimentales.

Universidad del País Vasco- Euskal Herriko Unibertsitatea. Vitoria-Gasteiz

\section{España}

Correspondencia: Javier Gasco. Escuela Universitaria de Magisterio de Vitoria-Gasteiz. Juan Ibañez de Santo Domingo, 1. Vitoria-Gasteiz, 01006 (Araba).E-mail: javier.gasco@ehu.es

C Education \& Psychology I+D+i and Ilustre Colegio Oficial de Psicología de Andalucía Oriental (Spain) 


\section{Resumen}

Introducción. La motivación constituye un factor importante en el aprendizaje de las matemáticas. Dentro de este ámbito educativo, la resolución de problemas ocupa un lugar central en la mayoría de currículos de matemáticas en Educación Secundaria. El objetivo de esta investigación es detectar las diferencias en motivación en función de las estrategias empleadas para la resolución de problemas aritmético-algebraicos.

Método. Se han analizado los procedimientos de resolución de tres problemas verbales aritmético-algebraicos y se han recopilado datos sobre la motivación mediante un cuestionario basado en el modelo de expectativa-valor de Eccles y sus colaboradores adaptado para la aplicación en el aprendizaje de las matemáticas. La muestra está compuesta por 598 estudiantes de $2^{\circ}, 3^{\circ}$ y $4^{\circ}$ de Educación Secundaria Obligatoria (ESO) de la Comunidad Autónoma Vasca.

Resultados. Los resultados obtenidos indican que el grupo de alumnos con perfil de resolución algebraico obtiene puntuaciones superiores tanto en valor de la tarea como en la autoeficacia percibida. Además, el grupo de resolución sin perfil definido declara que el estudio de las matemáticas supone una mayor pérdida de oportunidades para realizar otras actividades.

Discusión. Por una parte, el alumnado que resuelve los problemas algebraicamente muestra una motivación superior al grupo que alterna el álgebra y la aritmética como estrategias de resolución; por tanto, parece ser que la supuesta flexibilidad en la resolución implica menor motivación. Por otra parte, se discute la baja motivación del alumnado sin perfil de resolución definido en relación a su bajo rendimiento.

Palabras clave. Educación matemática, resolución de problemas, modelo motivacional de expectativa-valor, educación secundaria. 


\title{
The Motivation of Secondary School Students in Mathematical Word Problem Solving
}

\begin{abstract}
Introduction. Motivation is an important factor in the learning of mathematics. Within this area of education, word problem solving is central in most mathematics curricula of Secondary School. The objective of this research is to detect the differences in motivation in terms of the strategies used to solve word problems.

Method. It is analyzed the resolution procedures of three word problems. Furthermore, motivation data were collected through a questionnaire based on expectancy-value model of Eccles and her colleagues and adapted to the application in math learning. The sample consists of 598 students in 8th, 9th and 10th grade of Secondary Education in the Basque Autonomous Community.

Results. The results indicate that the group of students with algebraic resolution profile obtained higher scores in both task value and self-efficacy. In addition, the group without a define profile of resolution declares that the study of mathematics means greater loss of opportunity to do other activities.

Discussion. On the one hand, the students who solve problems algebraically shows a higher motivation comparing with the mixed resolution group; therefore, it appears that the alleged flexibility in resolution means less motivation. On the other hand, it is discussed the low motivation of students without a resolution profile in relation to their performance.
\end{abstract}

Keywords. maths education, word problem solving, motivational model of expectancyvalue, secondary education. 


\section{Introducción}

La investigación educativa en matemáticas reconoce la relevancia de los problemas aritmético-algebraicos. También es conocida la influencia de la motivación académica en todas las áreas de instrucción, incluida la enseñanza-aprendizaje de las matemáticas. A continuación se realiza una revisión de la investigación previa en ambos sentidos.

\section{La resolución de problemas aritmético-algebraicos}

En la sociedad de la información que impera hoy en día, el aprendizaje de las matemáticas se relaciona con la resolución de situaciones complejas y las competencias conceptuales que requieren habilidad verbal del alumnado (Sciarra y Seirup, 2008). Dichas aptitudes, principalmente adquiridas gracias al dominio de la resolución de problemas, fomentan el razonamiento verbal y constituyen la base de multitud de currículos y programas de estudios de matemáticas en todo el mundo.

Los problemas aritmético-algebraicos se definen como el conjunto que reúne a problemas que en el ámbito escolar se resuelven mediante el recurso a varias operaciones aritméticas elementales que se van combinando hasta obtener el resultado del problema (resolución aritmética), o bien mediante el planteamiento de ecuaciones que posteriormente se resuelven hasta obtener el resultado (resolución algebraica) (Cerdán, 2008). Por lo tanto, para encontrar el resultado de este tipo de problemas, se requiere del cálculo de expresiones aritméticas o de la resolución de ecuaciones.

La dicotomía entre calificar un problema de aritmético o algebraico representa una de las mayores controversias en al ámbito del álgebra educativa. Wagner y Kieran (1988) proponen una serie de preguntas que permiten acotar la naturaleza de los problemas y definir las dificultades que pueden aparecer en el tránsito de una resolución aritmética a una algebraica. Para Bednarz y Janvier (1996) los problemas aritméticos se definen como conectados ya que se puede fácilmente establecer una relación entre dos datos conocidos; en cambio, los algebraicos, se consideran como desconectados por no haber una conexión entre los datos conocidos. Cerdán (2008) afirma que es no es la 
estructura del problema lo que determina que sea aritmético o algebraico sino el proceso de traducción del enunciado verbal a las expresiones aritméticas o algebraicas.

La resolución de problemas matemáticos tiene un largo recorrido en los diferentes ciclos educativos; se comienza a aprender esta disciplina en Educación Primaria empleando operaciones aritméticas. El paso a Educación Secundaria conlleva la instrucción en una nueva técnica de resolución: el álgebra, procedimiento mucho más efectivo porque se puede aplicar a cualquier tipo de problema.

La transición de la aritmética al álgebra resulta compleja para el alumnado de Secundaria; el hecho de que ambas técnicas de resolución compartan símbolos como la suma, la resta, la multiplicación, la división y el signo igual complica el cambio (Kieran, 2007). Además, muchos de los problemas planteados en Secundaria no difieren mucho de los de Primaria, por no hacer demasiado complicada la resolución algebraica recién aprendida. (Stacey y MacGregor, 2000).

Diferentes estudios han detectado multitud de dificultades que el alumnado de Secundaria encuentra en su iniciación al aprendizaje del álgebra:

1. Las dificultades para operar con la incógnita (Herscovics y Linchevski, 1994; Filloy, Rojano, y Puig, 2008).

2. La tendencia a entender el signo igual como indicador de resultado (sentido operacional) y no como relación entre dos cantidades (sentido relacional) (Knuth, Alibali, Mcneil, Weinberg, y Stephens, 2005).

3. La dificultad para comprender el empleo de letras en la función de incógnita (Booth, 1984).

4. La complejidad que supone transformar el enunciado del problema a ecuaciones. Este error se ha asociado a la sintaxis del enunciado (Clement, 1982; Clement, Lochhead y Soloway, 1979; Fisher, Borchert, y Bassok, 2011). 


\section{El modelo motivacional de expectativa-valor}

La motivación ha sido uno de los constructos más investigados en el contexto educativo. Se ha estudiado una amplia gama de puntos de vista teóricos que tratan de explicar la motivación académica. Cada uno de ellos se ha centrado en dimensiones clave de la motivación, como, por ejemplo, la autoeficacia (Bandura, 1997), la expectativa y el valor de la tarea (Wigfield y Eccles, 2000), las metas de logro (Ames, 1992), el autoconcepto (Guay, Marsh, y Boivin, 2003) y las atribuciones (Skinner, Wellborn, y Connell, 1990).

La motivación relacionada con el aprendizaje de las matemáticas ha sido estudiada en numerosas ocasiones, tanto en la Enseñanza Secundaria (Ahmed, Van der Werf, Kuyper, y Minnaert, 2013; Phan, 2012), como en el ámbito universitario (Olani, Hoekstra, Harskamp, y van der Werf, 2011; Peters, 2013; Sciarra y Seirup, 2008). Sin embargo, la motivación es una variable psicoinstruccional compleja, tanto por la multitud de enfoques y mediadas que se le asocian, como por las variaciones que puede sufrir entre diferentes disciplinas educativas (Bong, 2001). En este sentido, algunas investigaciones afirman que ciertos indicadores de la motivación pueden ser más específicos en unos dominios que en otros. Por ejemplo, Green, Martin, y Marsh (2007) indican que el valor que se le asigna a la tarea en matemáticas por parte del alumnado presenta un alto grado de especificidad, mientras que otros como la ansiedad, muestran una tendencia más general entre distintas materias educativas. Esto sugiere que una evaluación de la motivación para las matemáticas, particularmente en aspectos tales como las creencias de valor, es más adecuada cuando se dirigiré al dominio específico en lugar de considerar la motivación general.

El rendimiento académico en matemáticas está influenciado en gran parte por la motivación y el compromiso del alumnado en el proceso de aprendizaje. Aunque cada una de estas teorías aporta una valiosa perspectiva a la comprensión de la motivación académica, la focalización en cada proceso, sin tener en cuenta los demás, puede suponer una limitación a la investigación (Bong, 1996).

Por tanto, no queda resulto si un modelo que mida la motivación general es capaz de reflejar la implicación del alumnado en matemáticas, o si habría que realizar 
una selección de indicadores que ofreciera una visión motivacional más fiable. Uno de los modelos más completos que ha estudiado la motivación para las matemáticas es la teoría de expectativa-valor (Wigfield y Eccles, 2000); este modelo ha demostrado que las creencias de valor y de expectativa de éxito están relacionadas con el esfuerzo en el aprendizaje de las matemáticas, con una mayor matriculación en esta disciplina y con el rendimiento.

El modelo de expectativa-valor ha tenido una gran influencia en la investigación sobre la motivación, lo que se ha traducido en gran cantidad de trabajos empíricos que corroboran tanto su utilidad como su validez (Eccles, 2005a; Wigfield y Eccles, 2000). Esta teoría motivacional argumenta que la elección, la persistencia y el rendimiento de los individuos se pueden explicar a través de la autoeficacia (o la percepción de la propia capacidad para la tarea) y el grado de valoración que da la persona a su propia actividad (Wigfield y Eccles, 2000).Las expectativas de éxito, por tanto, reflejan las creencias del individuo sobre su capacidad en un determinado dominio. Este componente es similar a la construcción de la autoeficacia en la teoría cognitiva social (Bandura, 1997). El alumnado con creencias de autoeficacia débiles puede verse afectado por dudas e incertidumbre, mientras que una alta autoeficacia promueve la seguridad y los sentimientos positivos hacia las propias habilidades.

El valor se ocupa de las cualidades percibidas en la tarea y de cómo estas percepciones influyen en el deseo del alumnado a la hora de realizarla. Asimismo, este factor explora el nivel de interés y utilidad percibida que una persona asocia con una tarea en particular. Cuatro son los componentes de valor en el modelo mencionado: la importancia, el interés, la utilidad y el coste. La importancia analiza la relevancia de hacer bien la tarea. El interés o motivación intrínseca se define como el grado en que una persona disfruta al realizar la tarea o su interés en el contenido de la misma (Wigfield y Eccles, 1992). El tercer elemento, la utilidad, hace referencia a la medida en la que una tarea resulta instrumental para alcanzar una meta futura (Pintrich y Schunk, 2002). El último componente es el coste, que se conceptualiza como los aspectos negativos asociados a la tarea, tales como el esfuerzo y la pérdida de oportunidades para realizar otras actividades. 
Los estudios previos apoyan la solidez de esta teoría motivacional mostrando que, tanto la expectativa como el valor, están directamente relacionados con el rendimiento académico y con la elección de líneas de estudio en el dominio específico de las matemáticas (Spinath, Spinath, Harlaar, y Plomin, 2006). Más precisamente, el rendimiento mantiene una relación más estrecha con las expectativas que con el valor (Steinmayr y Spinath 2009), mientras que es el valor el predictor más eficaz de la persistencia y de la conducta de elección del alumnado en lo que respecta a estudios futuros (Eccles, 2005b; Wigfield y Eccles, 1992).

Cabe destacar que, aunque sean numerosos las investigaciones que vinculan este modelo motivacional al estudio de las matemáticas, no se han encontrado evidencias que aclaren la relación entre las estrategias de resolución de problemas empleadas y la motivación para las matemáticas. Teniendo en cuenta tanto la escasez de estudios sobre el tema como la relevancia educativa de las variables en cuestión, el objetivo de esta investigación es analizar las diferencias en la motivación para las matemáticas en función de las estrategias empleadas para resolver problemas aritmético-algebraicos. El estudio focaliza su exploración en alumnado de Educación Secundaria Obligatoria (ESO).

Finalmente, el propósito general del estudio pretende contribuir al esclarecimiento de las vinculaciones entre la motivación y la instrucción matemática; una mejor comprensión de dicha conexión permitiría plantear acciones educativas tanto en el contexto escolar como en el familiar, siempre en pos de avanzar en la enseñanza y el aprendizaje de una materia tan importante.

\section{Método}

\section{Participantes}

La muestra se compone de 631 estudiantes de $2^{\circ}$ de la Educación Secundaria obligatoria (13-14 años), de $3^{\circ}$ de la ESO (14-15 años) y de $4^{\circ}$ de la ESO (15-16 años), de los cuales 292 eran mujeres y 242 hombres. Por motivos de errores u omisiones en los cuestionarios, la muestra final se ha reducido a 598 sujetos. La recogida de datos se ha realizado en 8 centros educativos pertenecientes a la Comunidad Autónoma Vasca, 
de los cuales 5 eran integrantes de la red pública y 3 de la red privada concertada. En la tabla 1 se presentan los datos descriptivos de la distribución muestral:

Tabla 1. Distribución de la muestra por cursos y sexos (frecuencias absolutas y porcentajes)

\begin{tabular}{|c|c|c|c|c|}
\hline & & \multicolumn{2}{|c|}{ SEXO } & \multirow[b]{2}{*}{ Total } \\
\hline \multirow{5}{*}{ 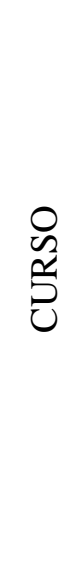 } & & Mujer & Hombre & \\
\hline & $2^{\circ} \mathrm{ESO}$ & 104 & 88 & $192(32.1 \%)$ \\
\hline & $3^{\circ} \mathrm{ESO}$ & 116 & 95 & $211(35.3 \%)$ \\
\hline & $4^{\circ} \mathrm{ESO}$ & 103 & 92 & $195(32.6 \%)$ \\
\hline & Total & $323(54 \%)$ & $275(46 \%)$ & $\mathrm{N}=598$ \\
\hline
\end{tabular}

\section{Instrumentos}

\section{Motivación para las matemáticas}

Con el fin de estudiar la motivación, se ha adoptado el modelo de expectativavalor. Por una parte, se mide la escala de expectativa de autoeficacia, para la cual los ítems se han seleccionado de la escala de autoeficacia del cuestionario MSLQ (Pintrich et al., 1991). Por otra parte, se evalúa la escala de valor de la tarea que está compuesta por cuatro categorías. Los componentes del valor de la tarea están definidos y evaluados por Eccles y sus colaboradores (Eccles et al., 1983; Eccles y Wigfield, 1995). Todos los ítems han sido adaptados por Berger y Karabenick (2011) para su aplicación en alumnado de matemáticas. Se ha empleado una traducción al castellano de dicho cuestionario (ver Anexo 1). 
Como recomiendan los autores del cuestionario original, se han agrupado los tres primeros componentes de valor (interés, utilidad e importancia) y se ha medido el coste por separado. De esta manera, los indicadores de motivación estudiados son tres: el valor, el coste y la expectativa de autoeficacia.

El cuestionario se estructura de la siguiente manera:

1) Expectativa de autoeficacia (3 ítems): Mide la creencia de autoeficacia que tiene el alumnado en cuanto al estudio de las matemáticas se refiere. Por ejemplo: "Estoy seguro de que puedo entender los contenidos más difíciles en matemáticas".

2) Valor de la tarea

Interés (3 ítems): Se refiere al interés o la predilección por las matemáticas. Por ejemplo: "Me gustan las matemáticas".

Utilidad (3 ítems): Contiene ítems relativos a la funcionalidad de las matemáticas en la vida real. Por ejemplo: "Creo que ser bueno en matemáticas puede ser útil en el futuro".

> Importancia (3 ítems): Mide el valor que pueden tener las matemáticas en la personalidad de cada cual. Por ejemplo:’Es importante para mí ser alguien que puede razonar utilizando fórmulas y operaciones matemáticas".

Coste (2 ítems): Tiene en cuenta el esfuerzo que supone el estudio de las matemáticas de cara a realizar otras actividades. Por ejemplo: "Tengo que dejar de hacer muchas cosas para aprender bien matemáticas".

El alumnado debe responder con arreglo a una escala tipo Likert de 5 puntos (donde $1=$ completamente en desacuerdo y $5=$ completamente de acuerdo).

\section{Resolución de problemas}

Los tres problemas propuestos son introducidos por Stacey y MacGregor (2000) y van de menor a mayor complejidad en cuanto a su resolución con métodos no algebraicos. Con el objetivo de medir las estrategias llevadas a cabo en cada problema propuesto, la codificación se ordena en cuatro categorías propuestas por Khng y Lee (2009): 
1. Algebraica: Si el planteamiento está definido por una o más incógnitas y se resuelve mediante una o más ecuaciones, se considera algebraico.

2. Aritmética: Si tanto el planteamiento como la resolución se basa en una técnica aritmética, esto es, sin recurrir ni a incógnita ni a ecuación, se tipifica como aritmética.

3. Mixta: Es la categoría donde se ubican los problemas en los que se usa una letra en alguna parte de la resolución pero la técnica es predominantemente aritmética. Se consideran procedimientos aritméticos.

4. Sin estrategia/sin respuesta: Se refiere a los métodos sin identificar o a los problemas sin resolver.

A la hora de clasificar la resolución como aritmética, se han dado como válidas la resolución aritmética y la mixta.

El instrumento de medida para las estrategias de resolución de problemas aritmético-algebraicos consta de tres problemas verbales aritmético-algebraicos a resolver. A cada individuo de la muestra se le agrupa en una categoría de las tres siguientes:

- Grupo G3 (Grupo de resolución de perfil algebraico): Esta categoría corresponde a los sujetos que resuelven correctamente la totalidad de los problemas algebraicamente, o, en su defecto, resuelven dos algebraicamente y en el tercero hacen un planteamiento algebraico (planteando las incógnitas y la ecuación), aunque no den con la solución correcta por un error de cálculo, y no procedimental.

- Grupo G2 (Grupo de resolución de perfil mixto): En este grupo se aglutinan los individuos que utilizan estrategias tanto algebraicas como aritméticas, dependiendo del problema a resolver. Se incluye, por tanto, el alumnado que resuelve correctamente dos problemas algebraicamente y uno aritméticamente, o viceversa (dos aritméticamente y uno algebraicamente).

- Grupo G1 (Grupo de resolución de perfil no definido): A este grupo pertenece el alumnado que no encaja ni en G3 ni en G2; es decir, no resuelve sistemáticamente ni de modo algebraico ni de modo aritmético, 
ni tampoco alternando ambas técnicas. Se incluyen en este conjunto a los sujetos que obtienen en algún problema (en uno, en dos, o en los tres) la clasificación de sin estrategia/sin respuesta.

El grupo G3 pertenece a los individuos que resuelven los problemas algebraicamente. El grupo G2 lo componen individuos que resuelven de manera mixta, en algunas ocasiones aritméticamente y en otras algebraicamente. Por último, en el grupo G1 se incluyen los sujetos que no hacen uso del álgebra o lo hacen escasamente, y tampoco dominan las técnicas aritméticas. Esta categorización, que prima el control sobre el empleo del álgebra y no sobre el de la aritmética, se debe a que, desde los primeros cursos de la ESO, el objetivo es que los problemas verbales se resuelvan algebraicamente. Las técnicas aritméticas (o heurísticas) que, tras la Educación Primaria, todavía se enseñan, responden a la experiencia previa del alumnado (Khng y Lee, 2009) y deberían ser complementarias al aprendizaje del álgebra como motor de resolución.

La pertinencia de tomar en cuenta el Grupo G2 se debe a un estudio previo (Gasco y Villarroel, 2012) que indica que un gran número de sujetos usa la aritmética en problemas adecuados para ello y sin embargo emplea el álgebra para resolver otros de muy difícil desarrollo aritmético.

\section{Análisis estadístico}

Con el objetivo de estudiar las diferencias entre variables se realiza la prueba no paramétrica para k muestras independientes de Kruskal-Wallis. Para realizar los test post-hoc se ha empleado la prueba no paramétrica U de Mann-Withney. La razón de la elección de dichas pruebas no paramétricas es que los datos recogidos no cumplen los criterios ni de normalidad ni de homocedasticidad.

En las pruebas para el análisis de dos muestras independientes, para la U de Mann-Withney, se ha calculado el tamaño del efecto (effect size), denotado por el parámetro r (Field, 2009; Rosenthal, 1991). La interpretación del coeficiente $\mathrm{r}$ es la siguiente: $r=.10$, tamaño del efecto débil; $r=.30$, tamaño del efecto moderado; y a partir de $\mathrm{r}=.50$ tamaño del efecto fuerte ( $\mathrm{r}$ toma valores entre 0 y 1 ). 


\section{Resultados}

En la tabla 2 se muestra la distribución de los grupos de resolución en función del curso académico:

Tabla 2. Distribución de los grupos de resolución en función del curso académico (frecuencias absolutas y porcentajes)

\begin{tabular}{|c|c|c|c|c|c|}
\hline \multirow{6}{*}{$\begin{array}{l}\mathscr{0} \\
0 \\
\text { S. } \\
\stackrel{0}{0}\end{array}$} & \multicolumn{5}{|c|}{ CURSO } \\
\hline & & $2^{\circ} \mathrm{ESO}$ & $3^{\circ} \mathrm{ESO}$ & $4^{\circ} \mathrm{ESO}$ & Total \\
\hline & G1 & $105(54.7 \%)$ & $70(33.2 \%)$ & $62(31.8 \%)$ & $237(39.6 \%)$ \\
\hline & G2 & $30(15.6 \%)$ & $52(24.6 \%)$ & $17(8.7 \%)$ & $99(16.6 \%)$ \\
\hline & G3 & $57(29.7 \%)$ & $89(42.2 \%)$ & $116(59.5 \%)$ & $262(43.8 \%)$ \\
\hline & Total & $192(32.1 \%)$ & $211(35.3 \%)$ & $195(32.6 \%)$ & $\mathrm{N}=598$ \\
\hline
\end{tabular}

G1=Grupo perfil no definido; G2=Grupo perfil mixto; G3=Grupo perfil algebraico

En la tabla 3 se presenta el análisis que explora las diferencias en las escalas de motivación (valor, coste y autoeficacia) entre los tres grupos de resolución (G1, G2 y G3).

Tabla 3. Diferencias entre los grupos de resolución en las escalas de motivación

\begin{tabular}{|c|c|c|c|c|c|c|}
\hline \multirow[b]{2}{*}{ Escala } & \multirow[b]{2}{*}{ Grupo } & \multirow[b]{2}{*}{ Media } & \multirow[b]{2}{*}{$\begin{array}{c}\text { Desviación } \\
\text { Típica } \\
\end{array}$} & \multicolumn{3}{|c|}{ Prueba de Kruskal-Wallis } \\
\hline & & & & $\chi^{2}$ & $\mathrm{gl}$ & $\mathrm{p}$ \\
\hline \multirow{3}{*}{ Valor } & G1 & 1.90 & .67 & \multirow{3}{*}{364.78} & \multirow{3}{*}{2} & \multirow{3}{*}{.000} \\
\hline & $\mathrm{G} 2$ & 3.43 & .71 & & & \\
\hline & G3 & 3.74 & .62 & & & \\
\hline \multirow{3}{*}{ Coste } & G1 & 2.65 & 1.01 & \multirow{3}{*}{40.22} & \multirow{3}{*}{2} & \multirow{3}{*}{.000} \\
\hline & $\mathrm{G} 2$ & 2.22 & .99 & & & \\
\hline & G3 & 2.11 & .80 & & & \\
\hline
\end{tabular}




\begin{tabular}{ccccccc} 
& $\mathrm{G} 1$ & 1.65 & .75 & & & \\
Autoeficacia & $\mathrm{G} 2$ & 3.56 & .71 & 369.25 & 2 & .000 \\
& $\mathrm{G} 3$ & 3.85 & .72 & & & \\
\hline
\end{tabular}

G1=Grupo perfil no definido; G2=Grupo perfil mixto; G3=Grupo perfil algebraico

Se dan diferencias intergrupales estadísticamente significativas en las tres escalas analizadas $(p<.001)$. El valor se refiere al interés, utilidad e importancia que otorga el alumnado al aprendizaje de las matemáticas. El coste tiene en cuenta el esfuerzo que supone el estudio de las matemáticas de cara a realizar otras actividades. La autoeficacia mide la creencia de eficacia personal que tiene el alumnado en cuanto al estudio de las matemáticas se refiere.

Con el objetivo de precisar las diferencias dos a dos existentes entre los grupos de resolución, se procede a efectuar los correspondientes test post-hoc mediante el test U de Mann-Withney (parámetros z y p) y sus respectivos tamaños del efecto (parámetro r):

1) Diferencias entre $G 1$ y G2: Se encuentran diferencias estadísticamente significativas en las tres escalas de motivación, Valor $(\mathrm{z}=-12.55, p<.001, r=.68)$, Coste $(z=-3.48, p<.001, r=.19)$, Autoeficacia $(z=-13.13, p<.001, r=.71)$. El alumnado perteneciente al grupo de perfil mixto, G2, que resuelve alternando el álgebra y la aritmética, obtiene medias superiores al grupo de perfil no definido, G1, en valor y autoeficacia. Sin embargo, el G1 obtiene una media superior en la escala de coste. El tamaño del efecto, $\mathrm{r}$, es fuerte en el caso del valor y la autoeficacia $(r>.50)$ y entre débil y moderado en el caso del coste $(.10<r<.30)$; es decir, la variación tiene más peso en las dos primeras escalas.

2) Diferencias entre G2 y G3. Se dan diferencias estadísticamente significativas en las escalas de valor y autoeficacia pero no en la escala de coste: Valor $(\mathrm{z}=-4.08$, $p<.001, r=.22)$; Coste $(z=-.70, p>.05)$; Autoeficacia $(z=-3.63, p<.001, r=.19)$. El grupo de resolución de perfil algebraico, G3, obtiene medias superiores al grupo de resolución mixto, G2, en ambas categorías. El tamaño del efecto en las 
diferencias tanto en valor como en autoeficacia es entre débil y moderado $(.10<\mathrm{r}<.30)$.

3) Diferencias entre G1 y G3: Se dan diferencias estadísticamente significativas en las tres escalas de motivación: Valor $(z=-17.94, p<.001, r=.80)$, Coste $(z=-6.31$, $p<.001, r=.28)$, y Autoeficacia $(z=-17.93, p<.001, r=.80)$. El grupo de perfil algebraico, G3, obtiene puntuaciones superiores al grupo de perfil no definido, G1, en valor y autoeficacia. En cambio, El grupo G1 obtiene puntuaciones superiores en la escala de coste. El tamaño del efecto, representado por el parámetro $\mathrm{r}$, es fuerte en el caso del valor y la autoeficacia y moderado en el caso del coste, dado que en los primeros dos casos es de $r=.80$ y en el caso del coste de $r=.28$.

\section{Discusión}

Los resultados en la motivación para las matemáticas se dividen en dos bloques: el concerniente a las diferencias entre los grupos de resolución algebraico y mixto, y las variaciones entre estos últimos y el grupo sin perfil definido.

Los integrantes del grupo algebraico logran en valor y autoeficacia resultados superiores a los del grupo mixto. El alumnado que resuelve algebraicamente valora más la tarea matemática en el sentido de tener un mayor interés y otorgarle mayor utilidad e importancia. Además se percibe más competente en la materia. Estas afirmaciones resultan significativas por el hecho de que no se han encontrado investigaciones que relacionen el procedimiento de resolución de problemas y la motivación. El coste es la única escala en la que no se dan diferencias entre el grupo algebraico y el mixto. Por tanto, el grupo que emplea procedimientos tanto aritméticos como algebraicos no aprecia un esfuerzo mayor en el aprendizaje de las matemáticas que el alumnado que resuelve algebraicamente exclusivamente.

En vista de los resultados, los datos sustentan que el alumnado que emplea el método algebraico de resolución está más motivado en el aprendizaje de las matemáticas, al menos en lo que respecta al modelo motivacional de valor-expectativa. Recíprocamente, es posible que una mayor motivación en la materia de matemáticas 
incentive el uso de estrategias matemáticas más exhaustivas y abstractas. Por consiguiente, ejercitar y dominar el álgebra en Educación Secundaria puede acarrear implicaciones educativas de gran relevancia que van más allá de la mejora en la eficacia resolutoria.

El segundo resultado obtenido es el vinculado a las diferencias entre el grupo sin perfil definido y los grupos algebraico y mixto. Tanto el grupo de resolución algebraico como el mixto superan relevantemente al grupo sin perfil definido en valor y autoeficacia. Además, el alumnado perteneciente a los grupos de perfil algebraico y mixto declara un coste inferior en el aprendizaje de las matemáticas en comparación con el grupo de perfil no definido. Los conjuntos de estudiantes que resuelven o plantean correctamente la totalidad de los problemas planteados (grupos algebraico y mixto) se contraponen al grupo que no alcanza dicho logro. Consecuentemente, es posible que la razón fundamental de la diferencia con respecto al grupo sin perfil radique en el rendimiento académico en matemáticas.

En la sociedad de la información que impera hoy en día, el rendimiento en matemáticas condiciona la resolución de situaciones complejas y las habilidades conceptuales que requieren habilidad verbal del alumnado (Sciarra y Seirup, 2008). Dicha habilidad, principalmente adquirida gracias a la competencia en la resolución de problemas, fomenta el razonamiento verbal y constituye la base de multitud de currículos y programas de estudios de matemáticas en todo el mundo. En este sentido, se han detectado varias razones para centrarse en el rendimiento en matemáticas en la investigación educativa. Diversos estudios coinciden en que, en contraste con otras materias escolares, es más probable que el aprendizaje de las matemáticas ocurra en la escuela y, por tanto, que sean especialmente sensibles a la instrucción (Burkam, Ready, Lee y LoGerfo, 2004; Porter, 1989).

Volviendo al aspecto motivacional, se han comprobado correlaciones positivas de la autoeficacia con mayores niveles de rendimiento académico en el área de matemáticas (Schunk y Pajares, 2002; Zimmerman y Martínez-Pons, 1990). Concretamente, Pajares (1996) asocia una mayor competencia percibida para la resolución de problemas matemáticos con un rendimiento superior en esa área. Además, 
el alumnado rinde más y está más motivado para optar por tareas cada vez más difíciles cuando cree que tiene la capacidad de ejecutarlas con éxito (Bandura, 1994; Covington, 1984; Weiner, 1985).

El trabajo de Eccles, Wigfield y sus colaboradores, entre otros, ha hecho hincapié en la particular contribución hecha por las expectativas de autoeficacia y el valor de la tarea en referencia a los logros en diversas materias educativas, incluidas las matemáticas. Asimismo remarcan la capacidad predictiva del modelo motivacional en cuestión (Eccles, 1987, Eccles, Adler y Meece, 1984; Eccles, Wigfield, Harold y Blumenfeld, 1993; Wigfield, Eccles, MacIver, Reuman y Midgley, 1991).

La escala de coste, a pesar de haber sido poco estudiada, representa una variable significativa para el alumnado en lo respectivo al rendimiento. La creencia de que los costes asociados a una conducta son muy elevados puede inducir a no llevarla a cabo. Por otra parte, a medida que aumenta el coste subjetivo de una tarea disminuye su valor neto (Wigfield y Eccles, 1992, 2000; Eccles y Wigfield, 2002).

En vista de los resultados obtenidos, se puede afirmar que el alumnado que resuelve los problemas verbales aritmético-algebraicos mediante el método algebraico se destaca por el alto grado de motivación en la materia de matemáticas, concretamente en su valoración de la tarea y en sus expectativas de autoeficacia.

Finalmente, no se pueden obviar las limitaciones del presente estudio. Debido a que es la primera vez que se aplica el cuestionario empleado en versión castellana, sería muy interesante insistir en el mismo y poder ponerlo a prueba en una muestra más extensa. Esta ampliación podría ayudar a comprobar la idoneidad de su aplicación en los sistemas educativos de lengua castellana. No hay que olvidar que la propia aplicación de cuestionarios puede que distorsione parcialmente los datos obtenidos. Los resultados concernientes a la motivación para las matemáticas han sido recopilados mediante autoinforme, con los consiguientes riesgos que eso supone para la credibilidad de los mismos. En un futuro, se podrían plantear entrevistas personales y/o observación de acciones concretas en el aula con el fin de conocer más estrechamente la influencia de esta variable psicoinstruccional. 


\section{Referencias}

Ahmed, W., Van der Werf, G., Kuyper, H. y Minnaert, A. (2013). Emotions, selfregulated learning, and achievement in mathematics: A growth curve analysis. Journal of educational psychology, 105(1), 150-161. doi:10.1037/a0030160

Ames, C. (1992). Classrooms: Goals, structures, and student motivation. Journal of Educational Psychology, 84, 261-271. doi:10.1037/0022-0663.84.3.261

Bandura, A. (1994). Self-efficacy. En V. S. Ramachaudran (Ed.), Encyclopedia of human behaviour, Vol. 4 (pp. 71-81). New York: Academic press. doi: 10.1037/10522-094

Bandura, A. (1997). Self-efficacy: The exercise of control. New York: Freeman.

Bednarz, N. y Janvier, B. (1996). Emergence and development of algebra as a problem solving tool: continuities and discontinuities with arithmetic. En N. Bednarz, C.Kieran \& L. Lee (Eds.), Approaches to Algebra (pp. 115-136). Dordrecht, The Netherlands: Kluwer Academic Publishers. doi:10.1007/978-94-009-1732-3_8

Berger, J. y Karabenick, S.A. (2011). Motivation and student's use of learning strategies: Evidence of unidirectional effects in mathematics classroom. Learning and Instruction, 21(3), 416-428.

Bong, M. (1996). Problems in academic motivation research and advantages and disadvantages of their solutions. Contemporary Educational Psychology, 21, 149165. doi:10.1006/ceps.1996.0013

Bong, M. (2001). Between- and within-domain relations of academic motivation among middle and high school students: Self-efficacy, task-value, and achievement goals. Journal of Educational Psychology, 93(1), 23-34. doi:10.1037/0022-0663.93.1.23

Booth, L. R. (1984). Algebra: children's strategies and errors. A report of the strategies and errors in secondary mathematics project. Windsor, United Kingdom: NFERNELSON.

Burkam, D., Ready, D., Lee, V. E. y LoGerfo, L. (2004). Social class differences in summer learning between kindergarten and first grade: Model specification and estimation. Sociology of Education, 77(1), 1-31. doi: 10.1177/003804070407700101

Cerdán, F. (2008). Estudios sobre la familia de problemas aritmético-algebraicos. Tesis doctoral no publicada. Universidad de Valencia. 
Clement, J. J. (1982). Algebra word problem solutions: Thought processes underlying a common misconception. Journal for Research in Mathematics Education, 13, 1630.

Clement, J. J., Lochhead, J. y Soloway, E. (1980). Positive effects of computer programming on students' understanding of variables and equations. Proceedings of the ACM 1980 Annual Conference (pp. 467-474). Nashville, TN: ACM.

Covington, M. V. (1984). The self-work theory of achievement motivation: Findings and implications. The Elementary School Journal, 18, 39-61.

Eccles, J. S. (1987). Gender roles and achievement patterns: An expectancy value perspective. En J. M. Reinisch, L. A. Rosenblum, y S. A. Sanders (Eds.), Masculinity/femininity: Basic perspectives (pp. 240-280). New York: Oxford University press.

Eccles, J. S. (2005a). Studying gender and ethnic differences in participation in math, physical science, and information technology. New Directions in Child and Adolescent Development, 110, 7-14. doi:10.1002/cd.146

Eccles, J. S. (2005b). Subjective task-value and the Eccles et al. model of achievementrelated choices. En A. J. Elliot y C. S. Dweck (Eds.), Handbook of competence and motivation (pp. 105-121). New York, NY: Guilford.

Eccles, J. S., Adler, T. F., Futterman, R., Goff, S. B., Kaczala, C. M., Meece, J. L. et al. (1983). Expectancies, values, and academic behaviors. En J. T. Spence (Ed.), Achievement and achievement motivation (pp. 75-146). San Francisco, CA: Freeman.

Eccles, J. S., Adler, T. F. y Meece, J. L. (1984). Sex differences in achievement: A test of alternative theories. Journal of Personality and Social Psychology, 46, 26-43.

Eccles, J. S. y Wigfield, A. (1995). In the mind of the actor: the structure of adolescent's achievement task values and expectancy-related beliefs. Personality and Social Psychology Bulletin, 21(3), 215-225.

Eccles, J. S. y Wigfield, A. (2002). Motivational beliefs, values and goals. Annual Review of Psychology, 53, 109-132.

Eccles, J. S., Wigfield, A., Harold, R. y Blumenfeld, P. B. (1993). Age and gender differences in children's self and task-perceptions during elementary school. Child Development, 64, 830-847. doi:10.2307/1131221

Field, A. (2009). Non-parametric tests. En Discovering statistics using SPSS (pp. 539583, $3^{\text {a }}$ edición). London: Sage. 
Filloy, E., Rojano,T. y Puig, L. (2008). Educational algebra. A theoretical an empirical approach. New York: Springer.

Fisher, K. J., Borchert, K. y Bassok, M. (2011). Following the standard form: Effects of equation format on algebraic modeling. Memory \& Cognition, 39, 502-515. doi: $10.3758 / \mathrm{s} 13421-010-0031-6$

Gascó, J. y Villarroel, T. (2012). Algebraic problem solving and learning strategies in compulsory secondary education. Procedia-Social and Behavioral Sciences, 46, 612-616.doi:10.1016/j.sbspro.2012.05.172

Green, J., Martin, A. J. y Marsh, H. W. (2007). Motivation and engagement in English, mathematics and science high school subjects: Towards an understanding of multidimensional domain specificity. Learning and Individual Differences, 17(3), 269-279. doi:10.1016/j.lindif.2006.12.003

Guay, F., Marsh, H. W. y Boivin, M. (2003). Academic self-concept and academic achievement: Developmental perspectives on their causal ordering. Journal of Educational Psychology, 95(1), 124-136. doi:10.1037/0022-0663.95.1.124

Herscovics, N. y Linchevski, L. (1994). A cognitive gap between arithmetic and algebra. Educational Studies in Mathematics, 27, 59-78. doi: 10.1007/BF01284528

Khng, K. H y Lee, K. (2009). Inhibition interference from prior knowledge: Arithmetic intrusion in algebra word problem solving. Learning and Individual Differences, 19, 262-268. doi: 10.1016/j.lindif.2009.01.004

Kieran, C. (2007). Learning and teaching algebra at the middle school through college levels: Building meaning for symbols and their manipulation. In F. K. Lester (Ed.), Second Handbook of Research on Mathematics Teaching and Learning (pp. 707-762). Reston, VA: National Council of Teachers of Mathematics.

Knuth, E. J., Alibali, M. W., Mcneil, N. M., Weinberg, A., y Stephens, A. C. (2005). Middle school students understanding of core algebraic concepts: equivalence and variable. ZDM, 37(1), 68-76. doi:10.1007/978-3-642-17735-4_15

Olani, A., Hoekstra, R., Harskamp, E. y van der Werf, G. (2011). Capacidad de razonamiento estadístico, auto-eficacia y creencias de valores en un curso universitario de estadística. Electronic Journal of Research in Educational Psychology, 9(1), 49-72. 
Pajares, F. (1996). Self-efficacy beliefs and mathematical problem solving of gifted students. Contemporary Educational Psychology, 21, 325-344. doi: 10.1006/ceps.1996.0025

Phan, H.P. (2012). Una exploración de metas de logro en el aprendizaje: un enfoque cuasi-cuantitativo. Electronic Journal of Research in Educational Psychology $10(2), 505-544$.

Extraido de:

http://www.investigacion/http://psicopedagogica.org/revista/new/buscador.php

Peters, M. L. (2013). Examining the relationships among classroom climate, selfefficacy and achievement in undergraduate mathematics: a multi-level analysis. International Journal of Science and Mathematics Education, 11(2), 459-480. doi: 10.1007/s10763-012-9347-y

Pintrich, P. y Schunk, D. (2002). Motivation in education. Theory, research and applications ( $2^{\text {nd }}$ edition). Prentice-Hall Merrill, Upper Saddle River, NJ.

Pintrich, P. R., Smith, D. A., García, T. y McKeachie, W. J. (1991). A Manual for the Use of the Motivational Strategies for Learning Questionnaire (MSLQ). AnnArbor, MINCRIPTAL: The University of Michigan.

Extraido de: http://search.proquest.com/docview/62892620?accountid=17248

Porter, A. (1989). A curriculum out of balance: The case of elementary school mathematics. Educational Researcher, 18(5), 9-15.

Rosenthal, R. (1991). Meta-analytic procedures for social research (2a edición). Newbury Park, CA:Sage.

Schunk, D. H. y Pajares, F. (2002). The development of academic self-efficacy. En A. Wigfield, y J. S. Eccles (Eds.), Development of achievement motivation: A volume in the educational psychology series (pp. 15-31). San Diego, CA: Academic Press.

Sciarra, D. T. y Seirup, H. J. (2008). The multidimensionality of school engagement and math achievement among racial groups. Professional School Counseling, 11(4), 218-228. doi:10.5330/PSC.n.2010-11.218

Skinner, E.A., Wellborn, J.G. y Connell, J.P. (1990). What it takes to do well in school and whether I've got it: A process model of perceived control and children's engagement and achievement in school. Journal of Educational Psychology, 82, 22-32. 
Spinath, B., Spinath, F. M., Harlaar, N., y Plomin, R. (2006). Predicting school achievement from general cognitive ability, self-perceived ability, and intrinsic value. Intelligence, 34(4), 363-374. doi:10.1016/j.intell.2005.11.004

Stacey, K. y MacGregor M. (2000). Learning the algebraic method of solving problems. Journal of Mathematical Behaviour, 18(2), 149-167. doi:10.1016/S07323123(99)00026-7

Steele, C. M. (1998). A threat in the air: how stereotypes shape intellectual identity and performance. American Psychologist, 52, 613-629. doi:10.1037//0003066X.52.6.613

Steinmayr, R. y Spinath, B. (2009). The importance of motivation as a predictor of school achievement. Learning and Individual Differences, 19(1), 80-90. doi: 10.1016/j.lindif.2008.05.004

Wagner, S. y Kieran, C. (1988). An Agenda for Research on the Learning and Teaching of Algebra. In S. Wagner y C. Kieran (Eds.), Research Issues in the Learning and Teaching of Algebra (pp. 220-237). Reston, VA: Lawrence Erlbaum Associates and National Council of Teachers of Mathematics.

Weiner, B. (1985). An attributional theory of achievement motivation an emotion. Psychological Review, 92, 548-573. doi:10.1037//0033-295X.92.4.548

Wigfield, A. y Eccles, J. S. (1992). The development of achievement tasks values: A theoretical analysis. Developmental Review, 12, 265-310. doi:10.1016/02732297(92)90011-P

Wigfield, A. y Eccles, J. S. (2000). Expectancy-value theory of achievement motivation. Contemporary Educational Psychology, 25, 68-81. doi: 10.1006/ceps.1999.1015

Wigfield, A., Eccles, J.S., Mac Iver, D.J., Reuman, D.A. y Midgley, C. (1991). Transitions during early adolescence: Changes in children's domains-specific selfperceptions and general self-esteem across the transition to junior high school. Developmental Psychology, 27, 552-565. doi: 10.1037//0012-1649.27.4.552

Zimmerman, B. J. y Martínez-Pons, M. (1990). Student differences in self-regulated learning: Relating grade, sex and giftedness to self efficacy and strategy use. Journal of Educational Psychology, 82, 51-59. doi: 10.1037//0022-0663.82.1.51 


\section{Anexos}

Anexo 1:

Cuestionario de motivación para las matemáticas (Traducción castellana del original de Berger y Karabenick, 2011):

Notas:

1) Todas las respuestas se ajustan a una escala Likert 5 (donde $1=$ Completamente en desacuerdo, 2=En desacuerdo, 3=Ni de acuerdo ni en desacuerdo, 4=De acuerdo y 5=Completamente de acuerdo).

2) El orden de los ítems expuesto no se ajusta con el empleado en la prueba llevada a cabo con la muestra.

Valor de la tarea

Interés

1. Me gustan las matemáticas.

2. Disfruto con las matemáticas.

3. Las matemáticas son emocionantes.

\section{Importancia}

1. Es importante para mí ser alguien que sea bueno en matemáticas.

2. Creo que ser bueno en matemáticas es parte importante de mi personalidad.

3. Es importante para mí ser alguien que puede razonar utilizando fórmulas y operaciones matemáticas.

\section{Utilidad}

1. Creo que las matemáticas pueden ser útiles en el futuro porque me pueden ayudar.

2. Creo que ser bueno en matemáticas puede ser útil en el futuro.

3. Creo que ser bueno en matemáticas puede ser útil para encontrar trabajo o para ir a la universidad. 
Coste

1. Tengo que dejar de hacer muchas cosas para aprender bien matemáticas.

2. Creo que el éxito en matemáticas requiere dejar otras actividades que me gustan.

\section{Expectativa de autoeficacia}

1. Creo que tendré una excelente nota en matemáticas.

2. Estoy seguro de que puedo entender los contenidos más difíciles en matemáticas.

3. Tengo confianza en que puedo aprender los conceptos básicos enseñados en matemáticas.

\section{Anexo 2:}

Problemas verbales aritmético-algebraicos (traducción castellana del original de Stacey y MacGregor, 2000)

1. Se reparten caramelos entre Jon y Maitane. A Jon le dan 5 caramelos más que a Maitane. En total hay 47 caramelos. ¿Cuántos caramelos le tocan a cada uno?

2. Un grupo de estudiantes hace un viaje de 3 días. La distancia recorrida el segundo día es de $85 \mathrm{~km}$ más que el primer día. La distancia recorrida el tercer día es de $125 \mathrm{~km}$ más que el primero. La distancia total es de $1410 \mathrm{~km}$. ¿Qué distancia se recorre cada día?

3. Descubre el número que si lo multiplico por 8 , resto 3 y divido por 3 , el resultado es el doble del número que había pensado. 